\title{
2. SYNOPSIS
}

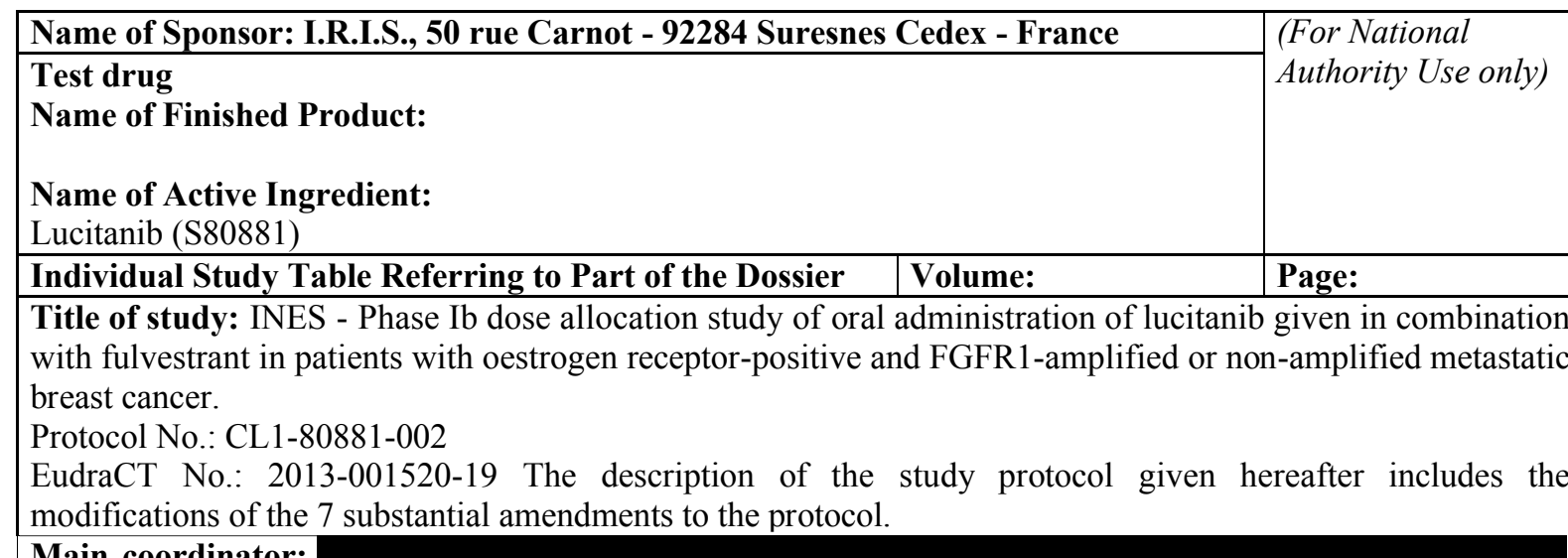

\section{Main coordinator:}

\section{Study centres:}

In all, 4 centres located in France included a total of 18 patients.

Publication (reference): Not applicable.

Studied period:
$\begin{aligned} & \text { Initiation date: } 10 \text { April } 2014 \text { (date of the first visit of the first } \\ & \text { patient) }\end{aligned}$ $\begin{aligned} & \text { Phase of development of the study: } \\ & \begin{array}{l}\text { Completion date: } 6 \text { March } 2017 \text { (date of last contact of the last } \\ \text { patient) }\end{array}\end{aligned}$

\section{Objectives:}

Primary objectives were to:

- Assess the tolerability of lucitanib in terms of Maximum Tolerated Dose (MTD) and Dose-Limiting Toxicities (DLTs) when administered with fulvestrant in patients with oEstrogen Receptor (ER) -positive, Human Epidermal Growth Factor Receptor 2 (HER2) negative, breast cancer after progression or recurrence on prior therapy (including prior therapy with fulvestrant).

- Identify the recommended Phase II dose (RP2D) of the oral capsule form of lucitanib given in combination with fulvestrant when administered in patients with oestrogen receptor-positive breast cancer after progression or recurrence on prior therapy (including prior therapy with fulvestrant).

\section{Secondary objectives were to:}

- Determine the pharmacokinetic (PK) profile of lucitanib and metabolites in combination with fulvestrant.

- Optional part: perform a pharmacogenomic (PG) analysis of inter-patients variation in genes encoding for proteins involved in absorption/distribution/metabolism/excretion (ADME).

- Measure tumour response to the oral capsule of lucitanib given in combination therapy with fulvestrant.

- Determine the pharmacodynamic (PD) profile of lucitanib:

- By characterising biological activity of lucitanib on soluble growth factors of interest.

- By characterising biological activity of lucitanib on tumour cells obtained from tumour biopsies before and on treatment (optional for allocation cohorts). As clarified by Amendment No. 2, the biopsies "before-treatment" became optional except for patients from expansion cohorts and the on-treatment biopsies became optional for all cohorts.

- By exploring biomarkers predictive for lucitanib response.

- Investigate any potential exposure dose-response relationships for safety, efficacy and PD.

The PK results (phase I part) are provided in Appendix 16.4. 


\section{Methodology:}

This was a multicentric, open, non-comparative, non-randomised, dose allocation (guided with a modified version of the Continual Reassessment Method i.e., mCRM) and dose expansion study, performed in menopausal women with ER+, HER2 negative, and Fibroblast Growth Factor Receptor 1 (FGFR1) amplified or non-amplified metastatic breast cancer.

This study was performed in strict accordance with Good Clinical Practice including the archiving of essential documents.

\section{Number of patients:}

Planned: up to 27 patients in the dose allocation part and up to 28 patients in the dose expansion part.

Included: 18 patients: 15 patients in the dose allocation part and 3 additional patients in the dose expansion part*.

* With the decision of 15 of September 2015 to complete the study by definitive stop of the recruitment, only three patients were included in dose expansion part.

\section{Diagnosis and main criteria for inclusion:}

Menopausal women (according to the NCCN definition), having histologically confirmed primary breast adenocarcinoma, with metastases (anatomic stage IV), whose tumour (either primary and/or metastases; metastases status mandatory for Part II) had significant expression of ER, absence of HER-2 overexpression or amplification, presence of FGFR1 amplification or not, and who had relapsed during or after treatment with fulvestrant.

Patients had to have adequate haematological, hepatic and renal function, estimated life expectancy $>12$ weeks and ECOG performance status $<2$, at study entry.

\section{Test drug:}

Lucitanib (Investigational Medicinal Product [IMP]; hard gelatin capsule of 2.5, 5 or $10 \mathrm{mg}$ ) was administered orally without food (at least 2 hours before or 2 hours after a meal), on a continuous once daily schedule of 28-day cycle in combination with Fulvestrant (Non Investigational Medicinal Product [NIMP]) administered at a dosage of $500 \mathrm{mg}$ ( 2 consecutive fulvestrant $250 \mathrm{mg}$ syringes in intra-muscular injections) once per cycle (28 days).

The first lucitanib dose was administered at the hospital, at C1D15 visit. Then the patient had to take lucitanib at home, approximately at the same time each day.

- Dose allocation - Part I: followed a modified version of the mCRM (with a target toxicity rate of 16 to $33 \%)$ to establish the MTD and the recommended dose (RD) of lucitanib for the expansion part when combined with fulvestrant. The study was conducted in cohorts of 3-6 patients, treated at increasing doses of lucitanib $(7.5 \mathrm{mg}, 10 \mathrm{mg}, 12.5 \mathrm{mg}$ or $15 \mathrm{mg})$ in combination with a fixed dose of fulvestrant $500 \mathrm{mg}$ monthly. Dose allocation could be considered only from the end of DLT assessment (from day 15 of cycle 2) and only after a meeting between the Sponsor, the investigators, and safety experts. Higher and/or lower doses of lucitanib could be proposed depending on available results during the study.

- Dose expansion - Part II: In this part, it was planned to open 2 cohorts: cohort A with FGF+ (receptor or ligand amplified) MBC patients and cohort B with FGF non-amplified MBC patients (14 patients planned for each cohort). Both cohorts were to be treated with lucitanib RD dose and fulvestrant $500 \mathrm{mg}$ monthly.

A the end of the dose allocation part, the RD of lucitanib (in combination with fulvestrant) for the expansion part was determined at $10 \mathrm{mg} /$ day* $^{*}$

Of note: only 3 patients were included in dose expansion part following the decision of 15 September 2015 to complete the study by definitive stop of the recruitment.

* The recommended dose of lucitanib (10 mg/day), given in combination with fulvestrant for Part II of the study was determined at the end of cohort meeting $N^{\circ} 3$ (10 June 2015).

\section{Comparator (Reference product and/or placebo):}

Not applicable.

\section{Duration of treatment:}

Each patient was to receive the combination of lucitanib with fulvestrant until unacceptable toxicity according to the investigator, disease progression or patient withdrawal.

The maximum number of cycles was at the discretion of the investigator. 


\section{Criteria for evaluation:}

Efficacy measurements:

Evaluation of antitumour activity was a secondary objective in this trial and was to be evaluated using the Response Evaluation Criteria in Solid Tumour (RECIST) version 1.1.

\section{Safety measurements:}

Determination of the MTD, DLTs (assessed during cycle 2 at C2D15) and the safety profile of lucitanib given in combination with fixed dose of fulvestrant were the primary objectives as well as RP2D establishment.

Safety measurements were the following:

- DLT assessment.

- Recording of Adverse Events (AEs). The toxicity was graded according to the Common Terminology Criteria for Adverse Events (CTCAE) version 4.0.

- Physical examination including vital signs (supine blood pressure, heart rate, respiratory rate and temperature), weight, and height (baseline only).

- ECOG performance status.

- Electrocardiogram (12-lead ECGs - central reading), and Left Ventricular Ejection Fraction (LVEF) assessed by echocardiogram or Multi Gated Acquisition (MUGA) scan.

- Laboratory examination: haematology, blood biochemistry (including Liver Function Tests, Kidney function tests, Thyroid Function Tests, troponin I) urinalysis and coagulation.

PK measurements: The plasma PK parameters of lucitanib (Cmax, Cmin and AUC) were measured.

\section{PD measurements:}

- Genomic (free circulating tumour DNA) measurements.

- Analysis of circulating blood proteins using ELISA and Mesoscale technologies (FGF-2, FGF-23, HIF-1 $\alpha$, M-CSF1, PDGF-AA, PDGF-BBs, VEGFR-1 and 2, VEGF-A -C -D, IL-6, IL-8, VCAM-1, Collagen IV, TIE-2 and PlGF), as well as biomarkers measured on tumour samples by IHC (FGFR1) and FISH (FGFR1 and the $11 \mathrm{q} 13$ amplicon containing FGF3, FGF4, FGF19 and CCND1).

- Characterisation of biological activity of lucitanib on tumour cells obtained from tumour biopsies before and on treatment (planned but not performed).

$P G$ measurements (optional samples):

Blood sample to interpret inter-patients PK variations in relation with polymorphisms of genes encoding for proteins involved in absorption/distribution/metabolism/excretion (ADME).

\section{Statistical methods:}

\section{Analysis Set:}

- Included Set (IS) (N = 18): All included patients.

- Safety Set (SS) $(\mathrm{N}=18)$ : Patients having taken at least one dose of Investigational Medicinal Product (IMP: lucitanib) or Non- Investigational Medicinal Product (NIMP: fulvestrant).

- Full Analysis Set (FAS) (N = 18): Included patients having taken at least one dose of IMP or NIMP.

- Response Evaluable Set (RES) $(\mathrm{N}=18)$ : All patients in the FAS having at least one baseline and one post-baseline tumour evaluation with at least one evaluable Overall Response (OR) (OR not equal to "non evaluable" or missing).

- DLT Evaluable Set (DLTES) $(\mathrm{N}=18)$ : All patients from SS who were evaluable for DLT according to the DLT assessment for the first 28 days of combination therapy (C1D15 to C2D15). A patient was not considered evaluable if:

- She did not receive at least $75 \%$ of lucitanib prescribed doses, unless treatment was stopped for DLT or

- She did not undergo a DLT assessment at C2D15 visit or

- She did not receive all fulvestrant prescribed doses from study entry to DLTs assessment visit (C2D15), unless treatment was stopped for a DLT. 


\begin{abstract}
Statistical methods: (Cont'd)
Efficacy analysis: was carried out on the FAS and RES.

The best overall response (BOR), the objective response rate (ORR) and the clinical benefit rate (CBR) as well as the duration of clinical benefit, the duration of response, the time to first response and the progression free survival (PFS) were provided in tables and/or graphs by dose level and overall. The OR was evaluated according to the investigator assessments. The survival functions of the time dependent parameters (duration of clinical benefit, the duration of response and the PFS) were estimated via Kaplan-Meier curves.
\end{abstract}

Study outcome: Descriptive statistics were provided in the IS, except for treatment duration and extent of exposure which were described in the SS, the FAS, and the RES as well as for concomitant treatments described only in the SS.

Safety analysis: Descriptive statistics were provided in the SS for each dose level and overall. DLTs were assessed in the DLTES.

\title{
PD analyses:
}

Circulating biomarkers were analysed in terms of value at C1D15 (baseline) and C2D1, and the description of change from baseline to C2D1 were provided by dose, by ORR status and overall. Comparison between C1D15 and C2D1 (only for overall) was studied using the paired Wilcoxon signed rank test and the Hodges-Lehmann's estimator for related sample.

Intratumoral biomarkers: FGFR1 (by FISH and IHC technologies) and 11q amplicon (by FISH technology) expression were graphically described according to the ORR status.

PK analysis: Individual and summary PK parameters of lucitanib (Cmax, Cmin and AUC) were provided.

\section{SUMMARY - CONCLUSIONS}

DISPOSITION OF PATIENTS AND BASELINE CHARACTERISTICS

Demographic and baseline characteristics of patients in the Included Set were in line with inclusion criteria defined for the study. Women enrolled in the study had median age of 66.0 years $(55.6 \%$ of them were aged within 65 and 84 years).

All the women included in the study had breast cancer of $E R+/ H E R 2$ - phenotype as requested by the protocol. Among them, $82.4 \%$ were also progesterone receptor positive.

Overall, the median disease duration from diagnosis was 13.9 years. At the time of inclusion, most of the patients $(16 / 18$ patients, $88.9 \%)$ had breast cancer for over 4 years. The overall median progression free interval (PFI i.e., interval between the start of last therapy and last progression) was 5.0 months. For most patients $(11 / 18$ patients, $61.1 \%)$, the time since latest progression was less or equal to 1 month. The overall median time since the first diagnosis of metastatic disease was of 3.5 years.

All the patients included in this study were previously treated by surgery, radiotherapy and drug treatment for their breast cancer. Patients received a median number of 3 previous drug treatment regimens for their breast cancer in metastatic setting.

As requested by the protocol, no patient was rated with an ECOG performance status $\geq 2$ and none was included with systolic blood pressure (SBP) $\geq 160 \mathrm{mmHg}$ and/or diastolic blood pressure (DBP) $\geq 100 \mathrm{mmHg}$. All patients with data available had QTcF below $450 \mathrm{~ms}$ and $\mathrm{LVEF} \geq 50 \%$.

\section{EXTENT OF EXPOSURE}

In this study, patients were treated at the dose of $10 \mathrm{mg} / \mathrm{day}(\mathrm{n}=12$ patients) and $12.5 \mathrm{mg} / \mathrm{day}(\mathrm{n}=6)$. In the Safety Set, the overall median global (i.e, lucitanib and fulvestrant) treatment duration was 28.1 weeks (24.6 weeks in the $10 \mathrm{mg}$ dose level and 44.6 weeks in the $12.5 \mathrm{mg}$ dose level). When focused on lucitanib, the median treatment duration was 25.9 weeks ( 22.2 weeks in the $10 \mathrm{mg}$ dose level and 39.1 weeks in the $12.5 \mathrm{mg}$ dose level). The median lucitanib relative dose intensity per patient was $77.4 \%$ (reflecting the treatment interruptions and dose reductions proposed for the management of toxicities described in the study protocol).

\section{EFFICACY RESULTS}

Overall, in the FAS, the BOR was Partial Response (PR) in 3/18 patients (16.7\%), while BOR was Stable Disease (SD) in $9 / 18$ patients (50.0\%), Non-Complete Response /Non-Progressive Disease (Non-CR/Non-PD) in $3 / 18$ patients $(16.7 \%)$ and Progressive Disease (PD) in $3 / 18$ patients $(16.7 \%)$.

Overall, the ORR was of $3 / 18$ patients (16.7\%). For these 3 patients, the time to first response was $8.1,15.3$ and 15.6 weeks and the duration of response was $20.1,56.1$ and 63.7 weeks, respectively.

The clinical benefit rate (CBR) was 55.6\% (10/18 patients). The overall median duration of clinical benefit was 39.6 weeks. 


\section{SUMMARY - CONCLUSIONS (Cont'd) EFFICACY RESULTS (Cont'd)}

Tumour evaluation during the treatment period - FAS $(N=18)$

\begin{tabular}{|c|c|c|c|c|}
\hline & & $\begin{array}{c}10 \mathrm{mg} \\
(\mathrm{N}=12)\end{array}$ & $\begin{array}{l}12.5 \mathrm{mg} \\
(\mathrm{N}=6)\end{array}$ & $\begin{array}{c}\text { All } \\
(N=18)\end{array}$ \\
\hline Objective Response Rate ${ }^{(1)}$ & $\begin{array}{l}\mathrm{n}(\%) \\
95 \% \mathrm{CI}^{(3)}\end{array}$ & $\begin{array}{c}1(8.3) \\
{[1.49 ; 35.39]}\end{array}$ & $\begin{array}{l}2(33.3) \\
{[9.68 ; 70.00]}\end{array}$ & $\begin{array}{l}3(16.7) \\
{[5.84 ; 39.22]}\end{array}$ \\
\hline Clinical Benefit Rate ${ }^{(2)}$ & $\begin{array}{l}\mathrm{n}(\%) \\
95 \% \mathrm{CI}^{(3)}\end{array}$ & $\begin{array}{c}4(33.3) \\
{[13.81 ; 60.94]}\end{array}$ & $\begin{array}{c}6(100) \\
{[60.97 ; 100.00]}\end{array}$ & $\begin{array}{c}10(55.6) \\
{[33.72 ; 75.44]}\end{array}$ \\
\hline \multicolumn{5}{|l|}{ Duration of clinical benefit (weeks) } \\
\hline $\begin{array}{l}\text { Number of censors } \\
\text { Alive without new treatment nor PD } \\
\text { Start of new anti-cancer therapy }\end{array}$ & $\begin{array}{l}\mathrm{n} \\
\mathrm{n}(\%)\end{array}$ & $\begin{array}{l}- \\
- \\
-\end{array}$ & $\begin{array}{c}2 \\
1(50.0) \\
1(50.0)\end{array}$ & $\begin{array}{c}2 \\
1(50.0) \\
1(50.0)\end{array}$ \\
\hline $\begin{array}{l}\text { Number of events } \\
\text { Progressive disease }\end{array}$ & $\begin{array}{l}\mathrm{n} \\
\mathrm{n}(\%) \\
\text { Median } \\
95 \% \text { CI } \\
\text { Min ; Max }\end{array}$ & $\begin{array}{c}4 \\
4(100) \\
28.1 \\
{[27.9 ; 32.7]} \\
27.9 ; 32.7\end{array}$ & $\begin{array}{c}4 \\
4(100) \\
71.3 \\
{[29.1 ; 79.1]} \\
29.1 ; 79.1\end{array}$ & $\begin{array}{c}8 \\
8(100) \\
39.6 \\
{[27.9 ; 79.1]} \\
27.9 ; 79.1\end{array}$ \\
\hline Time to first response (weeks) & $\begin{array}{l}\mathrm{n} \\
\text { Mean } \pm \text { SD } \\
\text { Median } \\
\text { Min; Max }\end{array}$ & $\begin{array}{c}1 \\
8.14 \pm . \\
8.14 \\
8.1 ; 8.1\end{array}$ & $\begin{array}{c}2 \\
15.43 \pm 0.20 \\
15.43 \\
15.3 ; 15.6\end{array}$ & $\begin{array}{c}3 \\
13.00 \pm 4.21 \\
15.29 \\
8.1 ; 15.6\end{array}$ \\
\hline \multicolumn{5}{|l|}{ Duration of response (weeks) } \\
\hline $\begin{array}{l}\text { Number of events } \\
\text { Progressive Disease (PD) }\end{array}$ & $\begin{array}{l}\text { Nobs } \\
\mathrm{n}(\%) \\
\text { Median } \\
95 \% \text { CI } \\
\text { Min; Max }\end{array}$ & $\begin{array}{c}1 \\
1(100) \\
20.1 \\
{[. ; . \cdot]} \\
20.1 ; 20.1\end{array}$ & $\begin{array}{c}2 \\
2(100) \\
59.9 \\
{[56.1 ; 63.7]} \\
56.1 ; 63.7\end{array}$ & $\begin{array}{c}3 \\
3(100) \\
56.1 \\
{[20.1 ; 63.7]} \\
20.1 ; 63.7\end{array}$ \\
\hline \multicolumn{5}{|l|}{ Progression free survival (weeks) } \\
\hline $\begin{array}{l}\text { Number of censors } \\
\text { Alive without new treatment nor PD } \\
\text { Start of new anti-cancer therapy } \\
\text { Number of events } \\
\text { Progressive disease }\end{array}$ & $\begin{array}{l}\mathrm{n} \\
\mathrm{n}(\%) \\
\mathrm{n}(\%) \\
\mathrm{n} \\
\mathrm{n}(\%) \\
\text { Median } \\
95 \% \text { CI } \\
\text { Min; Max }\end{array}$ & $\begin{array}{c}- \\
- \\
- \\
12 \\
12(100) \\
23.6 \\
{[7.6 ; 28.1]} \\
7.4 ; 32.7 \\
\end{array}$ & $\begin{array}{c}2 \\
1(50.0) \\
1(50.0) \\
4 \\
4(100) \\
71.3 \\
{[29.1 ; 79.1]} \\
29.1 ; 79.1 \\
\end{array}$ & $\begin{array}{c}2 \\
1(50.0) \\
1(50.0) \\
16 \\
16(100) \\
28.0 \\
{[15.7 ; 32.7]} \\
7.4 ; 79.1 \\
\end{array}$ \\
\hline
\end{tabular}

(1) Objective Response Rate (Best overall response $=$ CR or PR)

(2) Clinical Benefit Rate (Best overall response $=C R$ or PR or stabilization ( SD or Non CR /Non PD) $>24$ weeks or at the end of cycle 6)

The Wilson method was used to calculate the $95 \%$ Confidence interval (95\% CI) of the estimate

Circulating proteins (biomarkers) analyses on changes from baseline were available in 16/18 patients. From baseline to C2D1, a statistically significant high increase was observed for placental growth factor $(+358 \%$ of change compared to median at baseline, adjusted $p$-value $=0.0003$ ). In addition, statistically significant increase was observed for VEGF-A ( $+47 \%$ for both ELISA and Mesoscale measurements, adjusted p-values = 0.0003 and 0.0114 respectively), IL-8 $(+36 \%$, adjusted p-value $=0.0142)$ and FGF23 $(+30 \%$, adjusted p-value $=0.0047)$, while a trend towards an increase was observed for VEGF-D $(+11 \%$, adjusted p-value $=0.0439)$. Because there was too few patients in the dose level of $12.5 \mathrm{mg}(\mathrm{N}=6)$ and in the group of responders $(\mathrm{N}=3)$, no conclusion could be drawn regarding results by dose level or responders.

Overall in the FAS restricted to patients with an available biopsy $(\mathrm{N}=9)$, the chromosomal region $11 q 13$ (containing FGF3, FGF4, FGF19 and CCND1) was found to be amplified in 4 patients: one having PR as BOR and 3 having $\mathrm{SD}$ as BOR.

The FGFR1 gene was found to be amplified in one patient who had SD as BOR. In this patient, the 11q13 region was also amplified, and the FGFR1 expression level was high (global Hscore of 150) consistently with FGRFR1 amplification (FISH results).

A low FGFR1 expression (Hscore $<50$ ) was observed for all other patients having an available biopsy. 


\section{SUMMARY - CONCLUSIONS (Cont'd) \\ SAFETY RESULTS \\ Dose allocation, $M T D, R D$ and $P K$ finding}

DLTs were assessed during cycle 2 . The dosages tested were $10 \mathrm{mg} /$ day and $12.5 \mathrm{mg} / \mathrm{day}$. Only one DLT, hypertension with SBP $\geq 160 \mathrm{mmHg}$ or DBP $\geq 100 \mathrm{mmHg}$ not controlled to SBP $<160 \mathrm{mmHg}$ and DBP $<100 \mathrm{mmHg}$ by antihypertensive therapy within seven days after optimisation of antihypertensive therapy, was observed in one patient of the first cohort at $10 \mathrm{mg} /$ day. No other DLT was reported throughout the study.

Taking into account the DLT information from the 9 patients treated at $10 \mathrm{mg} /$ day and the 6 patients treated at $12.5 \mathrm{mg} /$ day of lucitanib in combination with fulvestrant during the Part I of the study, the mCRM recommended the dose of $15 \mathrm{mg} /$ day for the next cohort. However, a pooled analysis, done on data from 4 lucitanib studies, showed that $10 \mathrm{mg} /$ day would be a better tolerated dose (emergent AEs appeared to be less frequent at $10 \mathrm{mg}$, especially grade 3 hypertension). In agreement with these safety considerations, it was decided to stop the dose allocation (Part I of the study, with $\mathrm{N}=15$ patients) and to start the dose expansion part (Part II) at the RD dose of $10 \mathrm{mg} /$ day, determined at the end of cohort meeting $\mathrm{N}^{\circ} 3$. Three additional patients were included in Part II of the study.

\section{- Emergent adverse events}

Overall summary for Emergent Adverse Events (EAEs) in the Safety Set

\begin{tabular}{lcc}
\hline & & All \\
& $(\mathbf{N}=\mathbf{1 8})$ \\
\hline Patients having reported & $\mathrm{n}(\%)$ & $18(100)$ \\
at least one EAE & $\mathrm{n}(\%)$ & $18(100)$ \\
at least one lucitanib only-related EAE & $\mathrm{n}(\%)$ & $9(50.0)$ \\
at least one fulvestrant only-related EAE & $\mathrm{n}(\%)$ & $7(38.9)$ \\
at least one lucitanib and fulvestrant related EAE & $\mathrm{n}(\%)$ & $18(100)$ \\
at least one lucitanib \pm fulvestrant related EAE & $\mathrm{n}(\%)$ & $17(94.4)$ \\
at least one severe EAE & $\mathrm{n}(\%)$ & $8(44.4)$ \\
Patients having experienced & $\mathrm{n}(\%)$ & $5(27.8)$ \\
at least one serious EAE & $\mathrm{n}(\%)$ & \\
at least one lucitanib \pm fulvestrant related serious EAE & $\mathrm{n}(\%)$ & $3(16.7)$ \\
Patients with treatment withdrawal & $\mathrm{n}(\%)$ & $1(5.6)$ \\
due to an EAE & $\mathrm{n}(\%)$ & $1(5.6)$ \\
due to a serious EAE & $\mathrm{n}(\%)$ & \\
due to a lucitanib \pm fulvestrant related serious EAE & $\mathrm{n}(\%)$ & $1(5.6)$ \\
Patients who died & $\mathrm{n}(\%)$ & $2(11.1)$ \\
during the treatment period & & \\
during the follow-up period &
\end{tabular}

$N$ : Number of overall patients; $n$ : Number of patients in a category; $\%:(n / N) * 100$

During the study, all patients reported at least one EAE. The most frequent affected System Organ Classes (SOCs) were vascular disorders (17/18 patients, 94.4\%), gastrointestinal disorders (15/18 patients, 83.3\%), endocrine disorders $(14 / 18$ patients, $77.8 \%)$, general disorders and administration site conditions $(12 / 18$ patients, $66.7 \%)$ and investigations (12/18 patients, $66.7 \%)$.

Overall, the most frequently reported EAEs were hypertension (16/18 patients, 88.9\%), hypothyroidism $(14 / 18$ patients, $77.8 \%)$, diarrhoea ( $9 / 18$ patients, $50.0 \%)$, asthenia $(8 / 18$ patients, $44.4 \%)$, headache (7/18 patients, $38.9 \%$ ), nausea (7/18 patients, 38.9\%) and GGT increased (6/18 patients, 33.3\%). Most of the EAEs resolved (79.7\% of the total EAEs) at the time of the report.

During the treatment period, most of the patients (13/18 patients, $72.2 \%)$ experienced at least one EAE of grade 3 as worst grade, while $3 / 18$ patients $(16.7 \%)$ reported at least one EAE rated grade 4 as worst grade.

In addition, one patient reported 2 EAEs of grade 5 (i.e., leading to death): malignant neoplasm progression and hepatic encephalopathy, both considered as non-related to the study drug (i.e., not related to lucitanib \pm fulvestrant) but due to disease progression, according to the investigator.

Overall, 216 out of the 365 total EAEs (59.2\% of the EAEs) were considered by the investigators to be related to lucitanib or/and fulvestrant. Among them, 160 EAEs in 18 patients $(100 \%)$ were considered as related to lucitanib only, while 15 EAEs in $9 / 18$ patients $(50.0 \%)$ were considered as related to fulvestrant only, and 41 EAEs in $7 / 18$ patients (38.9\%) were considered as related to both lucitanib and fulvestrant. In all, a total of 201 EAEs reported in 18 patients $(100 \%)$ were considered as related to lucitanib \pm fulvestrant. 


\section{SUMMARY - CONCLUSIONS (Cont'd) \\ SAFETY RESULTS (Cont'd)}

The most common EAEs related to lucitanib \pm fulvestrant (reported in at least 5 patients) were hypertension (15/18 patients, $83.3 \%)$, hypothyroidism (14/18 patients, $77.8 \%)$, diarrhoea (7/18 patients, $38.9 \%)$, asthenia ( 7 EAEs in $7 / 18$ patients, $38.9 \%$ ), nausea ( 9 EAEs in $6 / 18$ patients, $33.3 \%$ ), decreased appetite (6 EAEs in 5/18 patients, $27.8 \%$ ) and proteinuria ( 5/18 patients, $27.8 \%$ ).

A total of $3 / 18$ patients $(16.7 \%)$ had 4 EAEs that led to premature treatment discontinuation: two patients reported a hypertension event (grade 3, non-serious - for both patients), and one patient reported a hypertensive crisis event (grade 4, serious) and a transient ischaemic attack event (grade 2, serious). All these events were considered as related to the study drug according to the investigator.

In all, $8 / 18$ patients $(44.4 \%)$ experienced a total of 24 emergent serious adverse events during the treatment period. Of them, 8 events in 5 patients were considered as related to lucitanib \pm fulvestrant (of which all were related to lucitanib only): hypertension, ejection fraction decreased and neutropenia (one patient each), hypertensive crisis, hypertension and generalised tonic-clonic seizure in one patient, transient ischaemic attack and hypertensive crisis in one patient.

In all, 3 patients died during the study, all due to disease progression. One patient died during the treatment period (EAE described above) while the 2 others died during the follow-up period.

\section{- Laboratory tests}

For biochemistry parameters, the most frequently observed emergent abnormal values non-gradable were high TSH (15/18 patients, $83.3 \%)$.

Among gradable biochemical parameters, the most frequently observed severe (graded $\geq 3$ ) emergent value was high GGT (6/18 patients, $33.3 \%)$.

For haematological parameters, the most frequently reported emergent abnormal values non-gradable were high haematocrit (8/18 patients, $44.4 \%)$. Among gradable parameters, $2 / 18$ patients $(11.1 \%)$ reported at least one severe emergent abnormal value: 1 patient for neutrophil decreased (grade 4) and one for low platelet (grade 3).

For coagulation parameters, emergent out-of-reference-range value was observed for low prothrombin time in one patient $(5.6 \%)$.

A total of $12 / 18$ patients $(66.7 \%)$ had a urinary test positive for proteins under treatment while patients were negative, non-significant positive or missing at baseline.

\section{- Other safety evaluation}

Most patients (12/18 patients, 66.7\%) had an ECOG performance status that remained $\leq 1$ as worst value during the treatment period. Concerning weight loss, the relative median change in weight from baseline to lowest value was of $-6.0 \%$.

Regarding blood pressures, the median changes from baseline to highest SBP and DBP values were $40.0 \mathrm{mmHg}$ and $20.5 \mathrm{mmHg}$, respectively. A total of $14 / 18$ patients $(77.8 \%)$ had a highest SBP value $\geq 140 \mathrm{mmHg}$ during the treatment period while the baseline value was $<140 \mathrm{mmHg}$ (or missing). For DBP, $12 / 18$ patients $(66.7 \%)$ had a highest value $\geq 90 \mathrm{mmHg}$ during the treatment period while the baseline value was $<90 \mathrm{mmHg}$ (or missing).

The median change in heart rate from baseline to highest value was $10.5 \mathrm{bpm}$. In all, $3 / 18$ patients $(16.7 \%)$ had a highest heart rate (HR) value $\geq 100 \mathrm{bpm}$ during the treatment period while the baseline value was between $[60,100[\mathrm{bpm}$.

Emergent ECG abnormality was considered as clinically significant in one patient (5.6\%) and was reported as an EAE "Bundle branch block right" of grade 1, neither serious nor related to the study drug. The patient recovered under treatment. Regarding QTc Fridericia interval (QTcF), 3/18 patients (16.7\%) experienced maximum QTcF prolongation during the treatment period between $451 \mathrm{~ms}$ and $480 \mathrm{~ms}$. Among patients having available data $(\mathrm{n}=16), 5$ patients $(31.3 \%)$ had a maximum increase of QTcF interval from baseline within 31 and $60 \mathrm{~ms}$.

In patients of the Safety Set having available data $(n=16)$, the median relative change from baseline to lowest LVEF value on treatment was $-4.8 \%$, ranging from $-35 \%$ to $+20 \%$. 


\section{CONCLUSION}

This study was a phase Ib dose allocation study to determine the safety profile and the recommended phase II dose (RP2D) of lucitanib in combination with fulvestrant in patients with ER-positive, HER2negative and FGFR1-amplified or non-amplified metastatic breast cancer.

During the study, it was decided (on 15 September 2015) to complete the study by definitive stop of the recruitment of patients, in the context of a limited benefit/risk ratio for the patients for 2 reasons. First, recent in vitro experiments performed at Servier showed that lucitanib had no impact on FGF2-induced resistance to ER antagonist (fulvestrant) in several ER+ breast cancer cell lines (FGFR1 amplified or not), indicating that one of the hypotheses emitted to explain its activity in combination with fulvestrant was not demonstrated. Secondly, as palbociclib/fulvestrant combination is likely to become the new standard of care in advanced HR+, HER- breast cancer that had relapsed or progressed during or prior endocrine therapy (based on a significant prolongation of the PFS with the combination versus placebo/fulvestrant in the recent PALOMA-3 study), the question of clinical relevance of the results produced through the INES study arose.

In this context, a total of 18 patients were treated. The lucitanib doses tested were $10 \mathrm{mg} / \mathrm{day}$ and $12.5 \mathrm{mg} /$ day. One patient experienced a DLT at $10 \mathrm{mg} / \mathrm{day}$, a hypertension event with SBP $\geq 160 \mathrm{mmHg}$ or DBP $\geq 100 \mathrm{mmHg}$ not controlled to $\mathrm{SBP}<160 \mathrm{mmHg}$ and $\mathrm{DBP}<100 \mathrm{mmHg}$ by antihypertensive therapy within seven days after optimisation of antihypertensive therapy. Based on safety data from pooled analysis of 4 lucitanib studies, the recommended dose of lucitanib (in combination with fulvestrant) for the expansion part of the study was determined at $10 \mathrm{mg} / \mathrm{day}$ (at the end of cohort meeting No. 3).

The most frequent emergent adverse event considered as related to lucitanib \pm fulvestrant was hypertension (15/18 patients) as similarly observed in the first-in-human study of lucitanib as a single-agent, followed by hypothyroidism (14/18 patients). Both of them were attributable to lucitanib only, according to the investigator.

The best overall response was confirmed partial response in $3 / 18$ patients $(16.7 \%)$ and stable disease in $9 / 18$ patients $(50.0 \%)$. The overall clinical benefit rate was $55.6 \%$ with a median duration of 39.6 weeks. The median progression free survival was 28.0 weeks. Regarding circulating blood proteins, observed modulation of biomarkers were consistent with lucitanib's mechanism of action, i.e. targeting VEGFRs (significant increase of PIGF, VEGF-A and -D levels) and FGFR1 (significant increase of FGF23 levels).

Date of the report: 04 August 2017

Version of the report: Final version. 